\title{
Local Randomness in Candidate One-Way Functions
}

\author{
H. Niederreiter ${ }^{1}$ and C.P. SChNorR ${ }^{2}$ \\ 1 Österreichische Akademie der Wissenschaften, Institut für \\ Informationsverarbeitung, Sonnenfelsgasse 19, A-1010 Wien, Austria \\ e-mail: niedeqiinfo.oear, ac,at \\ 2 Fachbereich Mathernatik/Informatik, Universität Frankfurt, Postfach 111932, \\ D-6000 Frankfurt/M., Germany \\ e-mail: schnorreinformatik.uni-frankfurt.de
}

\begin{abstract}
We call a distribution on $n$-bit strings $(\varepsilon, e)$-locally random, if for every choice of $e \leq n$ positions the induced distribution on $e$-bit strings is in the $L_{1}$-norm at most $\varepsilon$ away from the uniform distribution on $e$-bit strings. We establish local randomness in polynomial random number generators ( $R N G$ ) thal are candidate one-way functions. Let $N$ be a squarefree integer and let $f_{1}, \ldots, f_{\ell}$ be polynomials with coefficients in $\mathbb{Z}_{N}=\mathbb{Z} / N \mathbb{Z}$. We study the $R N G$ that stretches a random $x \in \mathbb{Z}_{N}$ into the sequence of least significant bits of $f_{1}(x), \ldots, f_{\ell}(x)$. We show that this $R N G$ provides local randomness if for every prime divisor $p$ of $N$ the polynomials $f_{1}, \ldots, f_{l}$ are linearly independent modulo the subspace of polynomials of degree $\leq 1$ in $Z_{p}[x]$. We also establish local randomness in polynomial random function generators. This yields candidates for cryptographic hash functions. The concept of local randomness in families of functions extends the concept of universal families of hash functions by CARTER and WEGMAN (1979). The proofs of our results rely on upper bounds for exponential sums.
\end{abstract}

\section{Introduction and Summary}

A major open problem in cryptography is to establish one-way functions. While we cannot prove one-wayness it makes sense to analyse candidate one-way functions and to prove properties of these functions that are useful in cryptographic applications. We call a distribution on $n$-bit strings $(\varepsilon, e)$-locally random if for every choice of $e \leq n$ positions the induced distribution on $e$-bit strings is in the $L_{1}$-norm at most $\epsilon$ away from the uniform distribution on $e$-bit strings. We prove $(\varepsilon, e)$-local randomness for large classes of candidate one-way functions and candidate cryptographic hash functions.

We show that $\ell$-tuples of polynomials $\left(f_{1}, \ldots, f_{\ell}\right) \in \mathbb{Z}_{N}[x]^{\ell}$ with fixed coefficients in $\mathbb{Z}_{N}$ and for arbitrary odd squarefree $N$ provide local randomness if for every prime divisor $p$ of $N$ the polynomials $f_{1}, \ldots, f_{\ell}$ are linearly independent modulo the subspace of polynomials of degree $\leq 1$ in $\mathbb{Z}_{p}[x]$. To give an example let $N$ be prime $N>2^{n}$, let $f_{1}, \ldots, f_{\ell} \in \mathbb{Z}_{N}[x]$ be any polynomials that 
are linearly independent modulo the subspace of polynomials of degree $\leq 1$ in $\mathbb{Z}_{N}[x]$. We prove in Corollary 2 that for random $x \in \mathbb{Z}_{N}$ the bit string

$$
\left(f _ { 1 } ( x ) \left[1, \ldots, f_{\ell}(x)[1)\right.\right.
$$

consisting of the parity bits $f_{i}(x)\left[1\right.$ of the residues $f_{i}(x) \bmod N$ in $[0, N-1]$ is $(\varepsilon, e)$-locally random provided that $\varepsilon, n, \ell$ and $e$ satisfy the inequality

(1) $2^{-n / 2}(2 n \log 2)^{e+1} 2 \ell \leq \varepsilon$,

where $\log$ denotes the natural logarithm. E.g. we can chonse $n \geq 64, \ell=$ $\left\lfloor 2^{n / 7}\right\rfloor, \varepsilon=2^{-n / 7}, e=\lfloor n /(7 \log n)\rfloor$. Our main result comprises the case that $N$ is an arbitrary odd squarefree integer, that the output contains several bits from each of the residues $f_{i}(x) \bmod N, i=1, \ldots, \ell$, and that $x$ is chosen to be random in a subinterval $[0, M-1]$ of $[0, N-1]$.

Note that the above function

(2) $[0, N-1] \ni x \mapsto\left(f_{1}(x)\left[1, \ldots, f_{\ell}(x)[1)\right.\right.$

is a candidate one-way function. No inversion algorithm is known that is polynomial time in $\min \left(\ell, \log _{2} N\right)$. So far the one-wayness of the function (2) has only be proved for random RSA-moduli $N$ and RSA-polynomials $f_{i}=x^{e^{i}}$ (see below) provided that the RSA-scheme is secure. It is however possible that this one-way function is more secure than the RSA-scheme. We are not aware of any inversion algorithm which for $\mathrm{RSA}$-moduli $N$ runs in time $\min \left(2^{\ell}, N\right)^{\circ(1)}$. On the other hand the RSA-scheme can be broken by factoring $N$ using only $\exp (\sqrt{\log N \log \log N})$ many steps. Is there any inversion algorithm that uses knowledge of the factorization of RSA-numbers $N$ ? Is there any inversion algorithm that uses the structure of particular odd moduli $N$ and of particular non-constant polynomials $f_{i}$ ? Of course the function (2) can easily be inverted for $N=2$ since $f_{i}(x)[1$ only depends on $x[1=x \bmod 2$. Also the problem of inverting is trivial for constant functions as $f_{i}(x)=x^{N-1}(\bmod N)$ with $N$ prime. Are there more exceptions? Almost nothing is known about the problem to invert (2). However if we cannot even find inverting algorithms for particular cases given the factorization of the modulus then this may be a sign that the function (2) is a truly one-way function.

It is important that the source of randomness in $\left(f_{1}(x)\left[1, \ldots, f_{\ell}(x)[1)\right.\right.$ is the random argument $x$ while the coefficients of $f_{1} \ldots, f_{l}$ are all fixed. Such functions are cryptographically interesting. A well known example is the random number generator (RNG) related to the RSA-scheme by ALEXI, CHOR, Goldreich and Schnorr (1988) and Micali, Schnorr (1991). E.g. let $N$ be the product of two large random primes and let the integer $e \geq 3$ be relatively prime to $\varphi(N)$. Then the mapping

$$
[0, N-1] \ni x \mapsto\left(x ^ { e } \left[1, x^{e^{2}}\left[1, \ldots, x^{\ell}[1)\right.\right.\right.
$$

where $x^{e^{i}}$ is taken modulo $\mathrm{N}$, is a perfect (in the sense of YAO (1982) and BLUM, MICALI (1982)) RNG provided that the RSA-scheme is secure. 
The functions $x \mapsto\left(f_{1}(x)\left[1, \ldots, f_{\ell}(x)[1)\right.\right.$ extend the class of polynomial random number generators (RNG) proposed by Micali and SChNorR (1991) which stretch a random seed $x \in\left[1, N 2^{-k}\right]$ into a polynomial residue $P(x)(\bmod N)$. Micali and Schnorr prove that the $m$ least significant bits of $P(x)(\bmod N)$ are in the $L_{1}$-norm at most $O\left(N^{-1 / 2} 2^{k+m}(\log N)^{2} \operatorname{deg}_{N}(P)\right)$ away from the uniform distribution provided that $N$ is prime and $\operatorname{deg}_{N}(P) \geq 2$ wherc $\operatorname{deg}_{N}(P)$ is the degree of $P$ when $P$ is considered modulo $N$.

So far local randomness has mainly been studied in functions that are easy to invert, see Alon, Babal, Itai (1986), Luby (1986), Schnorr (1988), MAURER, Massey (1989), NaOr, NaOr (1990), Nisan (1990) and Alon, Goldreich, Hastad, Peralta (1990). Most of these constructions are methodically simple and are not directed towards cryptographic applications. They aim at minimizing the number of random bits that are used in randomised algorithms. Merely the quadratic character construction by ALON et alii (1990) is similar to our generator, it relies on Weil's theorem. Our proof of local randomness relies on upper bounds for exponential sums and an inequality on quantitative Fourier inversion. Wc use upper bouuds for the discrepancy of polynomial residues from NiEderReiter (1977) and we extend these bounds from prime moduli to arbitrary squarefree moduli.

We also establish random function generators, associated with fixed polynomials, that provide local randomness. These generators are candidates for cryptographic hash functions. We associate with a polynomial $P \in \mathbb{Z}_{N}[x]$ of degree $d$ a polynomial function family $P_{z}(y)=P(z+y)$ where $z$ is the function name and $y$ is the input. For fixed $k, m \leq \log _{2} N$ we associate with a random $z \in \mathbb{Z}_{N}$ a random function

$$
P_{z}^{m}:\left[0,2^{k}-1\right] \longrightarrow\{0,1\}^{m}, y \longmapsto P(z+y)[m
$$

where $P(z+y)[m$ denotes the bit string consisting of the $m$ least significant bits of the residue $P(z+y) \bmod N$ in $[0, N-1]$.

We call a function family $\left\{P_{z}\right\}(\varepsilon, e)$-locally random if for random $z$ and for any $e$ distinct points $y_{1}, \ldots, y_{e}$ the distribution of the em-bit string $P_{z}\left(y_{1}\right) \ldots P_{z}\left(y_{e}\right)$ is in the $L_{1}$-norm at most $\varepsilon$ away from the uniform distribution on $e m$-bit strings.

We prove in Theorem 6 that the above family of functions $\left\{P_{z}^{m}\right\}$ is $(\varepsilon, e)$ locally random, if $N$ is prime, $d=\operatorname{deg} P$ satisfies $e+1 \leq d<N$ and if

$$
N^{-1 / 2}(\log N)^{e+1} 2^{e m+2} d \leq \varepsilon .
$$

A family of functions is an $e$-universal family of hash functions as introduced by CARTER and WEGMAN (1979) if and only if it is $(0, e)$-locally random. Our hash functions require fewer random bits than those of Carter and Wegman since 
we only randomize the input of the polynomial whereas Carter and Wegman randomize all its coefficients. The main point however is that our hash-functions are - if $\operatorname{deg} P$ is sufficiently large - candidates for cryptographically secure hashing whereas the Carter-Wegman hash functions are easy to invert. Thus for the first time we establish local randomness in families of cryptographic hash functions.

\section{Random Number Generators that Provide Statistical Local Randomness}

We present in Theorem 1 our main result and we derive from it $R N G$ 's that are locally random. In order to prove Theorem 1 we establish in Theorem 3 an upper bound on the discrepancy for multidimensional polynomial number sequences. This upper bound relies on an upper bound for exponential sums given in Lemma 4 and on an inequality of Niederreiter (1977) on quantitative Fourier inversion.

Notation. Let $p_{1}, \ldots, p_{r}$ be $r$ distinct primes, $N=p_{1} \cdots p_{r}$ (i.e. $N$ is squarefree) and $\mathbb{Z}_{N}=\mathbb{Z} / N \mathbb{Z}$. Let $\mathbf{F}=\left(f_{1}, \ldots, f_{\ell}\right)$ be an $\ell$-tuple of polynomials $f_{j} \in \mathbb{Z}[x], j=1, \ldots, \ell$. We denote by $d_{i}\left(f_{j}\right)$ the degree of $f_{j}$ when $f_{j}$ is considered $\bmod p_{i}$ and we put $d_{i}(\mathbf{F})=\max _{1 \leq j \leq \ell} d_{i}\left(f_{j}\right)$. We define $c_{i}(\mathbf{F})=\min \left(d_{i}(\mathbf{F})-1, \sqrt{p_{i}}\right)$ for $i=1, \ldots, r$ and $c(\mathbf{F}) \equiv \prod_{i=1}^{r}\left(c_{i}(\mathbf{F})+1\right)$. We call $\mathrm{F}$ N-admissible if for every prime divisor $p_{i}$ of $N$ the polynomials $f_{1}, \ldots, f_{\ell}$ are linearly independent modulo the subspace of polynomials of degree $\leq 1$ in $\mathbb{Z}_{p_{i}}[x]$. In this case we also call the set of polynomials $f_{1}, \ldots, f_{\ell}$ $N$-admissible. Thus $f_{1}, \ldots, f_{\ell}$ are $N$-admissible if for $i=1, \ldots, r$ and for all $a_{1}, \ldots, a_{\ell} \in \mathbb{Z}$ either the polynomial $\sum_{j=1}^{\ell} a_{j} f_{j}\left(\bmod p_{i}\right)$ is non-linear or $a_{1}=\cdots=a_{\ell}=0\left(\bmod p_{i}\right)$.

We let $\log N$ denote the natural logarithm of $N$. We identify $\mathbb{Z}_{N}$ with the integer interval $[0, N-1]$. We abbreviate the set $\{0,1\}^{n}$ as $I_{n}$ and we identify the integer interval $\left[0,2^{n}-1\right]$ with $I_{n}$. If $y \in[0, N-1]=\mathbb{Z}_{N}$ and $n \leq \log _{2} N$ we let $y\left[n \in I_{n}\right.$ denote the bit string consisting of the $n$ least significant bits of $y$. Let $\mathbb{N}$ denote the set of positive integers.

A collection of $m$ least significant output bits. We associate with $\mathbf{F}=$ $\left(f_{1}, \ldots, f_{\ell}\right) \in(\mathbb{Z}[x])^{\ell}, N \in \mathbb{N}$ and $\mathbf{m}=\left(m_{1}, \ldots, m_{\ell}\right) \in \mathbb{N}^{\ell}$ the mapping

$$
\mathbf{F}^{\mathbf{m}}:[0, N-1] \rightarrow I_{m}, \quad x \mapsto \prod_{j=1}^{\ell}\left(f_{j}(x)\left[m_{j}\right) \quad \text { with } \quad f_{j}(x) \in \mathbb{Z}_{N}\right.
$$

where $m=\sum_{j=1}^{\ell} m_{j}$ and $\Pi$ is the concatenation of strings. The mapping $F^{m}$ outputs a collection of $m$ least significant bits of $F(x)$, where $F(x)$ is taken modulo $\mathrm{N}$. 
Our main theorem provides explicit estimates for the max-norm difference between the distribution induced by $\mathbf{F}^{\mathbf{m}}(\boldsymbol{x})$ for random $x \in[0, M-1] \subset[0, N-1]$, $N$-admissible $\mathbf{F}$ and the uniform distribution on $\{0,1\}^{m}$.

Theorem 1. Let $N$ be odd and squarefree, let $\mathbf{F}, \mathbf{m}, m, \mathbf{F}^{\mathbf{m}}$ be as above and let $F$ be $N$-admissible. Then for $N>148,1 \leq M \leq N$ and random $x \in[0, M-1]$ we have that

$$
\max _{z \in\{0,1\}^{m}}\left|\operatorname{prob}\left[\mathbf{F}^{\mathbf{m}}(x)=z\right]-2^{-m}\right| \leq \frac{4}{M} \sqrt{N}(\log N)^{\prime+1} \mathbf{c}(\mathbf{F}) .
$$

The condition that $\mathbf{F}$ is $N$-admissible cannot be completely removed from Theorem 1 . Theorem 1 does not hold for linear polynomials $f_{1}, \ldots, f_{\ell}$ with $\ell \geq 2$. This is because the least significant bits in two linear polynomials are highly correlated. On the other hand our proof shows that Theorem 1 holds for a single polynomial of degree 1 in the case that $N=M$.

For example let $N>2^{512}$ be prime and let $d=2^{32}$. Then the polynomials $x^{2}, \ldots, x^{d}$ are $N$-admissible. Consider for random $x \in[0, N-1]$ the bit string $\left(x^{2}\left[1, \ldots, x^{d}[1) \in I_{d-1}\right.\right.$. For any choice of 24 bit positions $2 \leq i_{1}<i_{2} \ldots<$ $i_{24} \leq 2^{32}$ and every $z \in\{0,1\}^{24}$ we have that

$$
\mid \operatorname{prob}\left(x ^ { i _ { 1 } } \left[1 \cdots x^{i_{24}}[1=z)-2^{-24} \mid<2^{-44} .\right.\right.
$$

This follows from Theorem 1 with $\ell=24, f_{j}=x^{i j}$ for $j=1, \ldots, 24, N=M$ and $c(F) \leq 2^{32}$.

Definition. A random variable $y$ ranging over a finite set $S$ is called statistically random within $\varepsilon$ (in $S$ ) if $\sum_{s \in S}|\operatorname{prob}(y=s)-1 / \# S| \leq \varepsilon$, i.e. the $L_{1}$-norm statistical difference of $y$ from the uniform distribution on $S$ is at most $\varepsilon$.

Definition. A probability distribution $D$ on $I_{n}$ is called $(\varepsilon, e)$-locally random if for any sequence of positions $1 \leq j_{1}<j_{2}<\cdots<j_{e} \leq n$ the substring $\left(y_{j_{1}}, \ldots, y_{j_{e}}\right) \in I_{e}$ of a $D$-random string $y=\left(y_{1}, \ldots, y_{n}\right)$ is statistically randorn within $\varepsilon$.

Using Theorem 1 we can stretch a short random seed into a long bit string that is "locally random".

Corollary 2. Let $N=p_{1} \cdots p_{r}$ be a product of $r$ distinct odd primes, $1 \leq$ $M \leq N$ and $N>148$. Let $f_{1}, \ldots, f_{\ell} \in \mathbb{Z}[x]$ be polynomials of degree at most $d$ that are $N$-admissible. Then for random $x \in[0, M-1]$ the bit string $\left(f_{1}(x)\left[1, \ldots, f_{c}(x)[1) \in I_{\ell}\right.\right.$ with $f_{j}(x) \in \mathbb{Z}_{N}$ is $(\varepsilon, e)$-locally random with $\varepsilon=2 \frac{\sqrt{N}}{M}(2 \log N)^{e+1} d^{r}$ for $e=1, \ldots, \ell$. 
Proof. Let $1 \leq j_{1}<j_{2}<\cdots<j_{e} \leq \ell$ be any sequence of $e$ output bit positions. We apply Theorem 1 with $\overline{\mathbf{F}}=\left(f_{j_{1}}, \ldots, f_{j_{e}}\right), \mathbf{m}=(1, \ldots, 1) \in \mathbb{N}^{e}$ and $m=\ell=e$. The $L_{1}$-norm difference between the distribution induced by $F^{\mathbf{m}}(x) \in\{0,1\}^{e}$ and the uniform distribution is at most $2^{e}$-times the max-norm difference. We have $c(\mathbf{F}) \leq d^{r}$, and thus by Theorem $1 \mathbf{F}(x)$ is statistically random within $2 \frac{\sqrt{N}}{M}(2 \log N)^{e+1} d^{r}$.

The aiscrepancy $D_{M}^{(\ell)}=D_{M}^{(\ell)}\left(\mathbf{y}_{1}, \ldots, \mathbf{y}_{M}\right)$ of $M$ points $\mathbf{y}_{1}, \ldots, \mathbf{y}_{M} \in[0,1)^{\ell}$ is defined to be

$$
D_{M}^{(\ell)}\left(\mathbf{y}_{1}, \ldots, \mathbf{y}_{M}\right)=\sup _{\mathcal{I}}\left|F_{M}(\mathcal{I})-V(\mathcal{I})\right|
$$

where $\mathcal{I}$ ranges over all half open subintervals $\mathcal{I}$ of $[0,1)^{\ell}$, i.e.

$$
\mathcal{I}-\left\{\left(z_{1}, \ldots, z_{\ell}\right) \in[0,1)^{\ell} \mid a_{i} \leq z_{i}<b_{i} \text { for } i=1, \ldots, \ell\right\}
$$

with $0<a_{i}<b_{i} \leq 1$ for $i=1, \ldots, \ell . \quad V(\mathcal{I})$ is the volume of $\mathcal{I}$ and $F_{M}(\mathcal{I})=M^{-1} \#\left\{k \mid \bar{y}_{k} \in \mathcal{I}\right\}$.

The proof of Theorem 1 relies on the following upper bound for the discrepancy of multidimensional polynomial sequences. For a real number $a$ we let $\{a\}$ denote the residue of $a \bmod \mathbb{Z}$ in the real interval $[0,1)$.

Theorem 3. Let $N$ be squarefree and let $D_{M}^{(\ell)}$ be the discrepancy of the $M$ points $\left(\left\{\frac{f_{1}(k)}{N}\right\}, \ldots,\left\{\frac{\operatorname{se}(k)}{N}\right\}\right) \in[0,1)^{\ell}$ for $k=1, \ldots, M$. If $\mathbf{F}=\left(f_{1}, \ldots, f_{\ell}\right)$ is $N$-admissible then $D_{M}^{(\ell)} \leq \frac{4 B}{M} \sqrt{N}(\log N)^{\ell+1} c(\mathbf{F})$ for $1 \leq M \leq N$ and $N>148$. Here $B=\sqrt{2}$ if $N$ is even and $B=1$ if $N$ is odd.

The proof is based on a bound for exponential sums. For $f \in \mathbb{Z}[x]$ and $n \in \mathbb{N}$ define

$$
S(f, n)=\sum_{x=1}^{n} e\left(\frac{f(x)}{n}\right) \text { with } e(u)=e^{2 \pi \sqrt{-1} u} \text { for } u \in \mathbb{R} \text {. }
$$

The praofs for Lemmata 4 and 5 are omitted due to lack of space. They are contained in the complete paper that is to appear in Siam J. Computing.

Lemma 4. If $N=p_{1} \ldots p_{r}$ is squarefree, $B$ is as in Theorem 3, and $f \in \mathbb{Z}[x]$ is arbitrary, then $|S(f, N)| \leq B \sqrt{N} \prod_{i=1}^{r} c_{i}^{\prime}(f)$, where $c_{i}^{\prime}(f)=\sqrt{p_{i}}$ if $d_{i}(f)<1$ and $c_{i}^{\prime}(f)=\min \left(d_{i}(f)-1, \sqrt{p_{i}}\right)$ if $d_{i}(f) \geq 1$.

Lemma 5. Let $D_{M}^{(\ell)}$ be the discrepancy of the $M$ points $\mathbf{y}_{k} \in[0,1)^{\ell}$ for $k-1, \ldots, M$ and lct $D_{N}^{(\ell+1)}$ be the discrepancy of the $N$ points $\left(y_{k}, \frac{k-1}{N}\right)$ for $k=1, \ldots, N$. Then $D_{M}^{(\ell)} \leq \frac{N}{M} D_{N}^{(\ell+1)}$ for $1 \leq M \leq N$. 
Proof of Theorem 3. Let $N$ have $r$ distinct prime factors. Put $C_{\ell}(N)=$ $(-N / 2, N / 2]^{\ell} \cap \mathbb{Z}^{\ell}, C_{\ell}^{*}(N)=C_{\ell}(N) \backslash\{0\}$ (here we use, as in NiEderreiter $(1977)$, the interval $(-N / 2, N / 2]$ rather than $[0, N))$. For $\mathbf{h}=\left(h_{1}, \ldots, h_{\ell}\right) \in$ $C_{\ell}(N)$ we put

$$
r(\mathbf{h}, N)=\prod_{j=1}^{\ell} r\left(h_{j}, N\right) \text { with } r\left(h_{j}, N\right)= \begin{cases}1 & \text { if } h_{j}=0 \\ N \sin \frac{\pi\left|h_{j}\right|}{N} \text { if } h_{j} \neq 0 .\end{cases}
$$

By Lemma 2.2 of Niederreiter (1977), we get

(3) $D_{N}^{(\ell+1)} \leq \frac{\ell+1}{N}+\frac{1}{N} \sum_{\mathbf{h} \in C_{i+1}^{*}(N)} \frac{1}{r(\mathbf{h}, N)}\left|S\left(h_{1} f_{1}+\ldots+h_{\ell} f_{\ell}+h_{\ell+1} x, N\right)\right|$, where $\mathbf{h}=\left(h_{1}, \ldots, h_{\ell+1}\right)$ and $f_{1}, \ldots, f_{\ell} \in \mathbb{Z}[x]$. By Lemma 4

(4) $\left|S\left(h_{1} f_{1}+\ldots+h_{\ell} f_{\ell}+h_{\ell+1} x, N\right)\right| \leq B N^{1 / 2} \prod_{i=1}^{r} c_{i}^{\prime}\left(h_{1} f_{1}+\ldots+h_{\ell} f_{\ell}+h_{\ell+1} x\right)$.

If $\mathrm{h} \in C_{\ell+1}^{*}(N)$ with $\left(h_{1}, \ldots, h_{\ell}\right)=0$, then $c_{i}^{\prime}\left(h_{1} f_{1}+\ldots+h_{\ell} f_{\ell}+h_{\ell+1} x\right)=$ $c_{i}^{\prime}\left(h_{\ell+1} x\right)=0$ for some $i$, namely when $h_{\ell+1} \neq 0 \bmod p_{i}$, and so

$$
\prod_{i=1}^{r} c_{i}^{\prime}\left(h_{1} f_{1}+\ldots+h_{\ell} f_{\ell}+h_{\ell+1} x\right)=0 .
$$

Thus we only have to consider those $\mathrm{h} \in C_{\ell+1}^{*}(N)$ with $\left(h_{1}, \ldots, h_{\ell}\right) \neq 0$. We split up the set of $\left(h_{1}, \ldots, h_{\ell}\right) \in C_{\ell}^{*}(N)$ according to the set of $i$ 's for which $d_{i}\left(h_{1} f_{1}+\ldots+h_{\ell} f_{\ell}\right) \leq 1$. For $I \subseteq A_{r}:=\{1,2, \ldots, r\}$ we put $H(I)=$ $\left\{\left(h_{1}, \ldots, h_{\ell}\right) \in C_{\ell}^{*}(N): d_{i}\left(h_{1} f_{1}+\ldots+h_{\ell} f_{\ell}\right) \leq 1\right.$ if and only if $\left.i \in I\right\}$. If $\left(h_{1}, \ldots, h_{\ell}\right) \in H(I)$ and $i \in A_{r} \backslash$, then for any $h_{\ell+1} \in C_{1}(N)$ we have

$$
d_{i}\left(h_{1} f_{1}+\ldots+h_{\ell} f_{\ell}+h_{\ell+1} x\right)=d_{i}\left(h_{1} f_{1}+\ldots+h_{\ell} f_{\ell}\right) \geq 2 .
$$

Since $d_{i}\left(h_{1} f_{1}+\ldots+h_{\ell} f_{\ell}\right) \leq d_{i}(\boldsymbol{F})$, it follows that

$$
c_{i}^{\prime}\left(h_{1} f_{1}+\ldots+h_{\ell} f_{\ell}+h_{\ell+1} x\right) \leq c_{i}(\mathbf{F}) .
$$

Using the trivial bound $c_{i}^{\prime}(f) \leq p_{i}^{1 / 2}$, we obtain

$$
\prod_{i=1}^{r} c_{i}^{\prime}\left(h_{1} f_{1}+\ldots+h_{\ell} f_{\ell}+h_{\ell+1} x\right) \leq \prod_{i \in I} p_{i}^{1 / 2} \cdot \prod_{i \in A, \backslash I} c_{i}(\mathbf{F})
$$

for any $\left(h_{1}, \ldots, h_{\ell}\right) \in H(I)$ and $h_{\ell+1} \in C_{1}(N)$. Together with (3) and (4) this yields

$$
\begin{aligned}
D_{N}^{(\ell+1)} & \leq \frac{\ell+1}{N} \\
& +B N^{-1 / 2} \sum_{I \subseteq A_{r}} \prod_{i \in I} p_{i}^{1 / 2} \cdot \prod_{i \in A_{r} \backslash I} c_{i}(\mathbf{F}) \sum_{\mathbf{h} \in H(I)} \frac{1}{r(\mathbf{h}, N)} \sum_{h_{\ell+1} \in C_{1}(N)} \frac{1}{r\left(h_{\ell+1}, N\right)}
\end{aligned}
$$


Using the inequality

(5) $\sum_{h \in C_{i}^{*}(m)} \frac{1}{r(h, m)}<\frac{2}{\pi} \log m+\frac{2}{5}$ for $m \geq 2$

from Niederreiter $[14,(2.7)]$ this yields

$$
\begin{aligned}
D_{N}^{(\ell+1)} & <\frac{\ell+1}{N} \\
& +B N^{-1 / 2}\left(\frac{2}{\pi} \log N+\frac{7}{5}\right) \sum_{I \subseteq A_{r}} \prod_{i \in I} p_{i}^{1 / 2} \cdot \prod_{i \in A_{r} \backslash I} c_{i}(\mathbf{F}) \sum_{\mathbf{h} \in H(I)} \frac{1}{r(\mathbf{h}, N)} .
\end{aligned}
$$

By the assumption of the theorem $\left(f_{1}, \ldots, f_{l}\right)$ is $N$-admissible. Therefore if $\left(h_{1}, \ldots, h_{\ell}\right) \in H(I)$ we get $h_{k}=0 \bmod p_{i}$ for $i \in I$ and $1 \leq k \leq \ell$, thus $h_{k}=0 \bmod \prod_{i \in I} p_{i}$ for $1 \leq k \leq \ell$. Therefore with $L=\prod_{i \in I} p_{i}$ we obtain

$$
\begin{aligned}
\sum_{\mathbf{h} \in H(I)} \frac{1}{r(\mathbf{h}, N)} & \leq \sum_{\substack{\mathbf{h} \in C_{i}^{*}(N) \\
\mathbf{h}=0_{\text {modL }}}} \frac{1}{r(\mathbf{h}, N)}=\sum_{\mathbf{h} \in C_{\ell}(N / L)} \frac{1}{r(L \mathbf{h}, N)}-1 \\
& =\left(\sum_{h \in C_{1}(N / L)} \frac{1}{r(L h, N)}\right)^{\ell}-1 \\
& =\left(1+\sum_{h \in C_{\mathbf{i}}^{*}(N / L)} \frac{1}{r(L h, N)}\right)^{\prime}-1 \\
& =\left(1+\frac{1}{L} \sum_{h \in C_{\mathbf{i}}^{*}(N / L)} \frac{1}{r(h, N / L)}\right)^{\ell}-1 \\
& <\left(1+\frac{1}{L}\left(\frac{2}{\pi} \log \frac{N}{L}+\frac{2}{5}\right)\right)^{\ell}-1 \quad \text { (by the inequality (5)) } \\
& <\frac{\ell}{L}\left(\frac{2}{\pi} \log N+\frac{7}{5}\right)^{\ell},
\end{aligned}
$$

where we applied the mean-value theorem in the last step. It follows that

$$
\begin{aligned}
D_{N}^{(\ell+1)} & <\frac{\ell+1}{N}+B \ell N^{-1 / 2}\left(\frac{2}{\pi} \log N+\frac{7}{5}\right)^{\ell+1} \sum_{I \subseteq A_{r}} \prod_{i \in I} p_{i}^{-1 / 2} \cdot \prod_{i \in A_{r} \backslash I} c_{i}(\mathbf{F}) \\
& =\frac{\ell+1}{N}+B \ell N^{-1 / 2}\left(\frac{2}{\pi} \log N+\frac{7}{5}\right)^{\ell+1} \prod_{i=1}^{r}\left(c_{i}(\mathbf{F})+p_{i}^{-1 / 2}\right) \\
& <B N^{-1 / 2}(\log N)^{\ell+1} c(\mathbf{F})\left(\frac{\ell+1}{N^{1 / 2}(\log N)^{\ell+1}}+\ell\left(\frac{2}{\pi}+\frac{7}{5 \log N}\right)^{\ell+1}\right) \\
& <B N^{-1 / 2}(\log N)^{\ell+1} c(\mathbf{F})\left(\frac{1}{60}(\ell+1) 5^{-\ell}+\ell\left(\frac{2}{\pi}+\frac{7}{25}\right)^{\ell+1}\right)
\end{aligned}
$$




$$
<4 B N^{-1 / 2}(\log N)^{\ell+1} c(\mathbf{F})
$$

provided that $\log N \geq 5$, i.e. that $N>148$. Together with Lemma 5 we get the result of Theorem 3 .

Proof of Theorem 1 Let $N$ be an odd squarefree integer and $\bar{f}_{j} \in \mathbb{Z}[x]$ be polynomials such that $\bar{f}_{j}(x)=2^{-m_{j}} f_{j}(x)(\bmod N)$ for $j=1, \ldots, \ell$. Application of Theorem 3 to $\overline{\mathbf{F}}=\left(\bar{f}_{1}, \ldots, \bar{f}_{\ell}\right)$ shows that the discrepancy $\bar{D}_{M}^{(\ell)}$ of $\left(\left\{\bar{f}_{1}(k) / N\right\}, \ldots,\left\{\bar{f}_{\ell}(k) / N\right\}\right)$ for $k=1, \ldots, M$ satisfies

$$
\bar{D}_{M}^{(\ell)} \leq{ }_{M}^{4} \sqrt{N}(\log N)^{\ell+1} c(\mathbf{F})
$$

where we use that $c(\mathbf{F})=c(\overline{\mathbf{F}})$. We apply to this inequality the equivalence

$$
\begin{aligned}
& \left\{\bar{f}_{j}(x) / N\right\} \in\left[k_{j} 2^{-m_{j}},\left(k_{j}+1\right) 2^{-m_{j}}\right) \quad \Longleftrightarrow \\
& {\left[f_{j}(x)\right]_{N}=-k_{j} N\left(\bmod 2^{m_{j}}\right) \quad \text { for } \quad j=1, \ldots, \ell,}
\end{aligned}
$$

where $\left[f_{j}(x)\right]_{N}$ is the residue of $f_{j}(x) \bmod N$ in $[0, N-1]$, and $0 \leq k_{j}<2^{m_{j}}$. To see the equivalence we note that $\left\{\bar{f}_{j}(x) / N\right\} \in\left[k_{j} 2^{-m_{j}},\left(k_{j}+1\right) 2^{-m_{j}}\right)$ implies that there is an integer $y$ satisfying

$$
k_{j} N \leq y<\left(k_{j}+1\right) N, y=f_{j}(x) \bmod N, y=0 \bmod 2^{m_{j}},
$$

and thus $\left[f_{j}(x)\right]_{N}=-k_{j} N\left(\bmod 2^{m_{j}}\right)$. This proves one direction of the equivalence and the converse direction is an immediate consequence.

We see from the above inequality and the equivalence that for every $y \in$ $\{0,1\}^{m}$

$$
\left|\frac{1}{M} \#\left\{x \subset[1, M]: \mathbf{F}^{\mathbf{m}}(x)-y\right\}-\frac{1}{2^{m}}\right| \leq \frac{4}{M} \sqrt{N}(\log N)^{\ell+1} c(\mathbf{F}) .
$$

The above proof of Theorem 1 extends to the following larger class of functions $\mathbf{F}^{\mathbf{u}}$. Let the polynomials $f_{1}, \ldots, f_{\ell} \in \mathbb{Z}_{N}[x]$ be $N$-admissible and let $u_{1}, \ldots, u_{\ell}$ be integers that are relatively prime to $N, \mathbf{F}=\left(f_{1}, \ldots, f_{\ell}\right)$ and $\mathbf{u}=\left(u_{1}, \ldots, u_{\ell}\right)$. Define $\mathbf{F}^{\mathbf{u}}$ as

$$
\mathbf{F}^{\mathbf{u}}:[0, N-1] \ni x \mapsto\left(\left(f_{i}(x) \bmod N\right) \bmod u_{i} \mid \text { for } i=1, \ldots, \ell\right) .
$$

Corollary 6. For $N>148,1 \leq M \leq N$ and random $x \in[0, M-1]$ the maxnorm difference between the distribution induced by $\mathbf{k}^{\mathbf{u}}(x)$ and the uniform distribution on $\left[0, u_{1}-1\right] \times \cdots \times\left[0, u_{\ell}-1\right]$ is at most $\frac{4}{M} \sqrt{N}(\log N)^{\ell+1} c(\mathbf{F})$.

Theorem 1 deals with the particular case that the integers $u_{i}$ are powers of 2 . It is necessary that $u_{1}, \ldots, u_{\ell}$ are relatively prime to $N$. The proof of the Corollary uses the polynomials $\bar{f}_{j}=u_{j}^{-1} f_{j}(\bmod N)$ and thus requires a division by $u_{j}$ modulo $N$. 


\section{Random Function Generators that Provide Statistical Local Randomness}

Let $H_{k, \ell}=I_{\ell}^{I_{k}}=$ "the set of functions $f: I_{k} \rightarrow I_{\ell}$ ". A random function generator $F$ is an efficient algorithm that generates from names $x \in I_{n}$ a function $f_{z}=F(x, *) \in H_{k, \ell}$.

We call a probability distribution $D$ on $H_{k, \ell}(\varepsilon, e)$-locally random if for random $f, f \in \in_{D} H_{k, \ell}$, for any set of $e$ distinct inputs $y_{1}, \ldots, y_{e} \in I_{k}$ the concatenated output $f\left(y_{1}\right) f\left(y_{2}\right) \cdots f\left(y_{e}\right) \in I_{e \ell}$ is statistically random within $\varepsilon$.

The concept of $(\varepsilon, e)$-locally random distribution $D$ on $H_{k, \ell}$ extends the concept of universal hash functions of Carter and Wegman (1979). If $D$ is $(0, e)-$ locally random then for any distinct inputs $y_{1}, \ldots, y_{e} \in I_{k}$ the bit string $f\left(y_{1}\right) f\left(y_{2}\right) \cdots f\left(y_{e}\right) \in I_{e}$ is truly random, i.e. $D$ is the probability distribution of an e-universal family of hash functions in the sense of Carter and Wegman.

Carter and Wegman show how to generate an $e$-universal family of hash functions in $H_{k, k}$ from $k e$ randorn bits. Let $K=G F\left(2^{k}\right)$ be the field with $2^{k}$ elements. If $\left(a_{0}, \ldots, a_{e-1}\right) \in K^{e}$ is random then the polynomial $P=\sum_{i=0}^{e-1} a_{i} x^{i} \in$ $K[x]$ yields an $t$-universal family of hash functions in $H_{k, k}$.

Let $N$ be a prine and $P \in \mathbb{Z}_{N}[x]$ be a polynomial with coefficients in the field $\mathbb{Z}_{N}$. We associate with $P$ and $k, \ell \in \mathbb{N}, k, \ell \leq \log _{2} N$, the function

$$
P^{\ell}: \mathbb{Z}_{N} \times\left[0,2^{k}-1\right] \rightarrow I_{\ell},(z, y) \mapsto P(y+z)[\ell .
$$

Here we let $P(y+z)\left[\ell\right.$, for $\ell \leq \log _{2} N$, denote the bil string consisting of the $\ell$ least significant bits of the residue of $P(y+z) \bmod N$ that is in $\mathbb{Z}_{N}$. We let $P_{z}^{\ell}: I_{k} \rightarrow I_{\ell}$ denote the function $P^{\ell}(z, *)$.

Theorem 7. Let $N$ bc prime, $N>148, P \in \mathbb{Z}_{N}[x], k, \ell \leq \log _{2} N$, let $P_{z}^{\ell}: I_{k} \rightarrow I_{\ell}$ be as above and let $e+1 \leq \operatorname{deg} P<N$. Then far random $z \in \mathbb{Z}_{N}$ the family of functions $\left\{P_{z}^{\ell}\right\}$ is $(\varepsilon, e)$-locally random with $\varepsilon=$ $N^{-1 / 2}(\log N)^{e+1} 2^{e \ell+2} \operatorname{deg} P$.

Proof. Let $d=\operatorname{deg} P$, let $y_{1}, \ldots, y_{e} \in \mathbb{Z}_{N}$ be pairwise distinct and let $f_{i} \in \mathbb{Z}_{N}[x]$ be the polynomial $f_{i}(x)=P\left(y_{i}+x\right)$ for $i=1, \ldots, e$. We next show that the polynomials $f_{1}, \ldots, f_{e}$ are linearly independent modulo the subspace of polynomials of degree $\leq 1$ in $\mathbb{Z}_{N}[x]$. For suppose that there are $b_{1}, \ldots, b_{e} \in \mathbb{Z}_{N}$ such that

$$
\operatorname{deg}\left(\sum_{i=1}^{e} b_{i} P\left(y_{i}+x\right)\right) \leq 1 .
$$


Then for $j=d-e+1, \ldots, d$ the $j$-th derivative of this linear combination vanishes at $x=0$, hence

$$
\sum_{i=1}^{e} b_{i} P^{(j)}\left(y_{i}\right)=0 \quad \text { for } j=d-e+1, \ldots, d
$$

It is sufficient to prove that the coefficient matrix $\left[P^{(j)}\left(y_{i}\right)\right]_{\substack{d-1 \leq+1 \leq e \\ 1 \leq d \leq d}}$ is nonsingular since this implies that $b_{1}=\cdots=b_{e}=0$. Suppose that there exist $h_{d-e+1}, \ldots, h_{d} \in \mathbb{Z}_{N}$ such that

$$
\sum_{j=d-e+1}^{d} h_{j} P^{(j)}\left(y_{i}\right)=0 \text { for } 1 \leq i \leq e .
$$

Put $g(x)=\sum_{j=d-e+1}^{d} h_{j} P^{(j)}(x)$, then $g\left(y_{i}\right)=0$ for $1 \leq i \leq e$. Since $y_{1}, \ldots, y_{e}$ are distinct and $\operatorname{deg}(g) \leq d-(d-c+1)=e-1$ we have $g=0$, so

$$
\sum_{j=d-e+1}^{d} h_{j} P^{(j)}(x)=0 .
$$

Comparing coefficients of $x^{e-1}$ we get $h_{d-e+1}=0$ (the coefficient of $x^{e-1}$ in $P^{(d-e+1)}$ is nonzero since $\left.d<N\right)$. Continuing in this manner, we obtain $h_{d-e+1}=\cdots=h_{d}=0$.

Since $f_{1}, \ldots, f_{e}$ are linearly independent modulo $\mathbb{Z}_{N}+x \mathbb{Z}_{N}$ we can apply Theorem 1 to $\mathbf{F}=\left(f_{1}, \ldots, f_{e}\right)$. Since $\prod_{j=1}^{e} f_{j}(z)\left[\ell \in I_{e \ell}\right.$ the $m$ in Theorem 1 is $e \ell$. The $\ell$ in Theorem 1 is $e$. Hence $\prod_{j=1}^{e} P\left(y_{j}+z\right)\left[\ell \in I_{e \ell}\right.$ is statistically random within $\varepsilon=N^{-1 / 2}(\log N)^{e+1} d 2^{\ell \ell+2}$. Therefore $\left\{P_{z}^{\ell}\right\}$ is $(\varepsilon, e)$-locally random.

\section{References}

[1] Alexi, W., Chor, B., Goldreich, O. and Schnorr, C.P.: RSA and Rabin Functions: certain parts are as hard as the whole. SIAM J. Comput., 17, 2 (1988), pp. $194-208$.

[2] Alon, N., Babai, L. and Itai, A.: A fast and simple randomised parallel algorithm for the maximal independent set problem. J. of Alg. 7 (1986), pp. 567 583.

[3] Alon, N., Goldreich, O., Hastad, J. and Peralta, R.: Simple constructions of almost $k$-wise independent random variables. Proceedings of the 31st IEEE Symposium on Foundations of Computer Science (1990) pp. $544-552$.

[4] BluM, L, BuUM, M., and Snub, M.: A simple unpredictable pseudo-random number generator. SIAM J. Comput. 15 (1986), pp. $364-383$.

[5] BLUM, M. and MICALI, S.: How to generate cryptographically strong sequences of pseudo-random bits. Proceedings of the 23rd IEEE Symposium on Foundations of Computer Science, IEEE, New York (1982); also SIAM J. Comput. 13 (1984), pp. $850-864$. 
[6] Carlitz, L. and Uchiyama, S.: Bounds for exponential sums. Duke Math. J. 24, (1957), pp. $37-41$.

[7] Carter, L. and Wegman, M.: Universal hash functions. J. Comp. and Syst. Sci. 18, (1979) pp. 143 - 154.

[8] Goldreich, O., Goldwasser, S. and Micali, S.: How to Construct Random Functions. Proceedings of the 25th IEEE Symposium on Foundations of Computer Science, New York, (1984); also Journal ACM 33, 4 (1986), pp. 792-807.

[9] LIDL, R., and NEDerneiter, H.: Finite Fields. Reading: Addison-Wesley 1983.

[10] LUBY, M.: A simple parallel algorithm for the maximal independent set problem. SIAM J. Comput., 15 (1986), pp. 1036 - 1053.

[11] Maurer, U. M., and MAssey, J.L.: Perfect local randomness in pseudo-random sequences. Proceedings Crypto '89, Lecture Notca in Computer Science, Vol. 435, Springer-Verlag 1990, pp. 100-112.

[12] Mrcali, S. and SchNorR, C.P.: Efficient, perfect polynomial random number generators. J. of Cryptology 3, (1991), pp. $157-172$.

[13] NAOR, J. and NaOr, M.: Small-bias Probability Spaces: Efficient Constructions and Applications. Proceedings of the 22nd ACM Symposium on Theory of Computing (1990), pp. 213-223.

[14] NiederReiter, H.: Pseudo-random numbers and optimal coefficients. Advances in Math. 26, (1977) pp. $99-181$.

[15] NISAN, N.: Pseudorandom generators for space-bounded computation. Proceedings of the 22nd ACM Symposium on Theory of Computing (1990), pp. 204-208.

[16] SCHNORR, C.P.: On the construction of random number generators and random function generators. Proc. FUROCRYPT '88, Lecture Notes in Computer Science, Vol. 330, Springer-Verlag 1988, pp. 225-232.

[17] Wer, A.: On some exponential sums. Proc. Nat. Acad. Sci. USA 34, (1948), pp. $204-207$.

[18] YAO, A.C.: Theory and applications of trapdoor functions. Proceedings of the 23rd IEEE Symposium on Foundations of Computer Science, IEEE, New York (1982), pp. 80-91. 